Reprint from Appl. Phys A. 122 (2016) 241

http://dx.doi.org/10.1007/s00339-016-9724-4

\title{
Direct observation of the lattice sites of implanted manganese in silicon
}

\author{
D. J. Silva, ${ }^{1,2}$ U. Wahl, ${ }^{3}$ J. G. Correia, ${ }^{3}$ L. M. Amorim, ${ }^{2}$ S. \\ Decoster, ${ }^{2}$ M. R. da Silva, ${ }^{4}$ L. M. C. Pereira,${ }^{2}$ and J. P. Araújo ${ }^{1}$ \\ ${ }^{1}$ IFIMUP and IN-Institute of Nanoscience and Nanotechnology, \\ Departamento de Física e Astronomia da Faculdade de Ciências da Universidade do Porto, 4169-007 Porto, Portugal \\ ${ }^{2}$ Instituut voor Kern- en Stralingsfysica, KU Leuven, 3001 Leuven, Belgium \\ ${ }^{3}$ Centro de Ciências e Tecnologias Nucleares, Instituto Superior Técnico, \\ Universidade de Lisboa, 2686-953 Sacavém, Portugal \\ ${ }^{4}$ Centro de Física Nuclear da Universidade de Lisboa, 1649-003 Lisboa, Portugal
}

(Dated: April 1, 2015)

\begin{abstract}
Mn-doped Si has attracted significant interest in the context of dilute magnetic semiconductors. We investigated the lattice location of implanted Mn in silicon of different doping types $\left(n, n^{+}\right.$and $p^{+}$) in the highly dilute regime. Three different lattice sites were identified by means of emission channeling experiments: ideal substitutional sites; sites displaced from bond-centered towards substitutional sites and sites displaced from anti-bonding towards tetrahedral interstitial sites. For all doping types investigated, the substitutional fraction remained below $\sim 30 \%$. We discuss the origin of the observed lattice sites as well as the implications of such structures on the understanding of Mn-doped Si systems.
\end{abstract}

\section{INTRODUCTION}

While Mn-doped III-V semiconductors, such as (Ga,Mn)As and (In,Mn)As, have become model dilute magnetic semiconductors (DMS), ${ }^{1}$ Mn-doped group IV semiconductors ( $\mathrm{Si}$ and $\mathrm{Ge}$ ) have also been intensively investigated and are even regarded as potentially advantageous compared to other DMS systems. ${ }^{2}$ Although the questions of (i) substitutional versus interstitial occupancy, and (ii) dilution versus precipitation, have been addressed (e.g. Refs. 3 and 4), the lattice location and thermal stability of $\mathrm{Mn}$ in $\mathrm{Si}$ and Ge is still poorly understood. While complex interplay effects between substitutional and interstitial Mn have been theoretically predicted (e.g. Refs. 5-8), assessing them experimentally is extremely challenging. These difficulties result to a large extent from the difficulty to probe and control the Mn incorporation in different configurations: substitutional, interstitial and precipitates of different compounds and structures. The excessively low solubility of $\mathrm{Mn}^{9}$ combined with its high interstitial diffusivity (activation energy of $\sim 0.67-0.72 \mathrm{eV})^{10,11}$ results in the formation of precipitates, so that at higher Mn concentrations isolated $\mathrm{Mn}$ in interstitial or substitutional form is not easily obtained. An example of secondary phase segregation is the formation of $\mathrm{MnSi}_{1.7}$ silicides. ${ }^{12,13}$ Several methods involving the introduction of Mn by heterostructures, with a reduced formation of secondary phases, have been addressed recently. ${ }^{14,15}$ Since the substitutional incorporation of $\mathrm{Mn}$ requires the existence of vacancies, ion implantation, which creates such defects through radiation damage, has often been investigated as a potentially reproducible and controllable way of substitutional $\mathrm{Mn}$ doping. ${ }^{2,3,12,13,16}$ In that respect, a detailed study of the lattice sites occupied by implanted $\mathrm{Mn}$ in the phase-pure regime (dilute, randomly distributed $\mathrm{Mn}$ ) should clarify whether this is indeed the case. We have addressed these questions by applying the electron emission channeling technique (EC) from implanted radioactive ${ }^{56} \mathrm{Mn}$ probes to different doping types of Si single crystals. The lattice sites of ${ }^{56} \mathrm{Mn}$ were inferred from the angular dependence of the emission yield of its $\beta^{-}$particles along various axial and planar directions. ${ }^{17-20}$ As it turns out, even at low concentrations only a minority of $\mathrm{Mn}(<30 \%)$ occupies in fact ideal substitutional sites, while the large majority forms more complex structures with implantation defects or $p$-type dopants. Our results not only clearly illustrate the difficulties in using ion implantation as means of substitutional Mn doping, but also shed light on the general interaction of Mn with defects and impurities in Si.

\section{EXPERIMENT}

${ }^{56} \mathrm{Mn}$ was implanted in: $n$-type $\mathrm{Si}(n$-Si), at an energy of (i) $30 \mathrm{keV}$ and (ii) $60 \mathrm{keV}$; (iii) $n^{+}$-type $\mathrm{Si}\left(n^{+}-\mathrm{Si}\right)$, at an energy of $30 \mathrm{keV}$; and (iv) $p^{+}$-type $\mathrm{Si}\left(p^{+}-\mathrm{Si}\right)$, at an energy of $30 \mathrm{keV}$. All samples had $<111>$ oriented surfaces. The implantation took place at the on-line isotope separator facility ISOLDE at CERN, which provides mass separated beams of radioactive isotopes of Mn produced by inducing nuclear fission of uranium carbide targets with a $1.4 \mathrm{GeV}$ proton beam. ${ }^{56} \mathrm{Mn}$ implantations were carried out under a vacuum $<1 \times 10^{-5}$ mbar, and under an angle of $17^{\circ}$ from the surface normal to avoid channeling implantation. Specifications about the used samples and implantation parameters can be seen in table I. The ${ }^{56} \mathrm{Mn}$ profile as well as the number of vacancies were estimated using the SRIM code. ${ }^{21}$ For the 60 $\mathrm{keV}$ implantation, the profile can be approximated by a Gaussian centered at $544 \AA$ with a straggling of 212 $\AA$. Each implanted $\mathrm{Mn}$ creates $\sim 1000$ vacancies. For the $30 \mathrm{keV}$ implantation, the profile is centered at $302 \AA$ with a straggling of $123 \AA$. At this implantation energy, each ${ }^{56} \mathrm{Mn}$ produces $\sim 500$ vacancies (half the number of those at $60 \mathrm{keV}$ ). For both implantation energies, the 
vacancy concentration peak is located midway from the peak concentration of ${ }^{56} \mathrm{Mn}$ to the surface.

After implanting ${ }^{56} \mathrm{Mn}$, the samples were annealed in situ at several temperatures for $10 \mathrm{~min}$ and further measured at room temperature. The electron yield was measured in the vicinity of the $<110>,<211>,<100>$ and $<111\rangle$ crystallographic directions using a position and energy sensitive detector. ${ }^{22}$ The resulting twodimensional experimental patterns were further corrected due to the background from $\gamma$ particles and backscattered $\beta^{-}$particles. While the $\gamma$ contribution was measured by blocking the $\beta^{-}$particles by means of closing a valve in front of the detector, the contribution of backscattered $\beta^{-}$particles was estimated with the Monte Carlo GEANT4 code. $^{23}$

\section{RESULTS}

The two-dimensional experimental patterns were fitted by theoretical $\beta^{-}$emission yields for ${ }^{56} \mathrm{Mn}$ emitters, calculated with the manybeam formalism for electron channeling. ${ }^{22}$ A range of lattice sites were considered in the theoretical patterns covering the following relevant high symmetry sites: substitutional $(\mathrm{S})$, hexagonal $(\mathrm{H})$, tetrahedral (T), bond-centered (BC), anti-bonding (AB), split (SP) and the so-called DS, DT, Y and C sites. Sites displaced from these high symmetry sites along $<111>$, $<100>$ and $<110>$ were also considered. The minimum step of displacement was $\sim 0.03 \AA$. These lattice sites are discussed in detail in Ref. 24.

The fitting procedure was started by allowing only for a variable fraction of emitter atoms on one type of lattice site. The minimization of the $\chi^{2}$ of fit resulted consistently, over the whole range of annealing temperatures, on sites displaced from $\mathrm{BC}$ towards $\mathrm{S}$ sites (near-BC sites), for the $n$ - and $n^{+}-\mathrm{Si}$ samples. For $p^{+}-\mathrm{Si}$, sites displaced from AB towards $\mathrm{T}$ sites (near-AB sites) presented the best $\chi^{2}$ of fit. The introduction of a second fraction, near-AB in the $n$ - and $n^{+}$-Si experiments and near-BC in the $p^{+}-\mathrm{Si}$ experiment, leads to an additional considerable decrease of the $\chi^{2}$ of fit. Since substitutional sites are recurrently mentioned in the literature we tested the ideal $\mathrm{S}$ site as a third fraction. It gave a further significant reduction of the $\chi^{2}$ of fit for all the annealing temperatures. The addition of a fourth lattice site did not result in a significant change in the $\chi^{2}$ of fit. Figure 1 shows the two-dimensional experimental patterns for ${ }^{56} \mathrm{Mn}$ emitters from the $n$-Si sample after implanting at $60 \mathrm{keV}$ and from the $n-, n^{+}$and $p^{+-} \mathrm{Si}$ samples after implanting at $30 \mathrm{keV}$. The dependence of the fraction on each identified lattice site as well as the respective sum for all the four experiments are plotted in Fig. 2. Finally, the dependence of the displacement of near-BC and near-AB sites with annealing temperature is shown in Fig. 3. In the following, we describe in detail the obtained results for each of the four experiments.

\section{A. $n$-type \\ 1. $30 \mathrm{keV}$}

After implanting at $30 \mathrm{keV}$, the fractions of the identified three lattice sites of ${ }^{56} \mathrm{Mn}$ are quite similar $(30 \%$ of ${ }^{56} \mathrm{Mn}$ occupies near-BC sites, $20 \%$ ideal S sites and $27 \%$ near-AB sites). This fact can be easily concluded from the prominent channeling $\{111\}$ planes indicative of the presence of sites near the BC position, prominent channeling $\{110\}$ planes characteristic of sites near $\mathrm{T}$ or $\mathrm{AB}$ positions and presence of the channeling peak along the $<211>$ direction originated by ideal $\mathrm{S}$ sites. The fitting results agree with this scenario for all annealing steps. In particular, after the $200^{\circ} \mathrm{C}$ anneal, $42 \%$ of ${ }^{56} \mathrm{Mn}$ occupies near-BC sites, $25 \%$ ideal S sites and $32 \%$ near-AB sites. The sum of all fractions varies with the annealing temperature. While a maximum is obtained at $T_{A}=200^{\circ} \mathrm{C}$, a reduction of the sum is prominent up to the annealing temperature of $700^{\circ} \mathrm{C}$, which is indicative that long-range diffusion, where Mn probe atoms move towards the bulk or reach the surface of the sample, starts already around $300^{\circ} \mathrm{C}$. The displacements of near-BC and near-AB sites are also similar for the different annealing temperatures. While near-BC sites are displaced $\sim 0.3 \AA$ from the ideal $\mathrm{BC}$ site, near- $\mathrm{AB}$ sites show a displacement of $\sim 0.3 \AA$ from the ideal AB site.

\section{2. $60 \mathrm{keV}$}

The best fit results showed that near-BC sites unambiguously prevail for all the annealing steps. In particular, after annealing at $200^{\circ} \mathrm{C}, 25 \%$ of ${ }^{56} \mathrm{Mn}$ occupies ideal $\mathrm{S}$ sites, $29 \%$ near-AB sites and $63 \%$ near-BC sites. The average displacement of near-BC and near-AB sites are $\sim 0.4 \AA$ from the ideal $\mathrm{BC}$ site and $\sim 0.5 \AA$ from the ideal $\mathrm{AB}$ site, respectively. Long-range diffusion of $\mathrm{Mn}$ seems also to start around $300^{\circ} \mathrm{C}$.

\section{B. $n^{+}$-type}

For highly-doped $n^{+}$-Si, we obtained similar twodimensional experimental patterns as in the case of lowdoped $n$-Si (from Fig. 1 (b) to $(\mathbf{c})$ ). Two differences are, however, observed in the evolution of the fractions and displacements with annealing temperature. First, from Fig. 2 (c) one can notice that the sum of all the fractions only starts to decrease after the $600^{\circ} \mathrm{C}$ anneal, while in $n$-Si the sum already reduces at $\sim 300^{\circ} \mathrm{C}$. There is, therefore, a higher thermal stability of the three observed lattice sites in $n^{+}-\mathrm{Si}$. Secondly, Fig. 3 shows that, compared to $n$-Si, near-AB sites are closer to the ideal $\mathrm{AB}$ site (average displacement of $\sim 0.1 \AA$ from the ideal $\mathrm{AB}$ site), while near-BC sites are closer to the ideal $\mathrm{BC}$ site (average displacement of $\sim 0.2 \AA$ from the ideal $\mathrm{BC}$ 
TABLE I. Sample and implantation details of the four experiments carried out in the present investigation.

\begin{tabular}{|c|c|c|c|c|c|c|}
\hline experiment & $\begin{array}{l}\text { doping } \\
\text { type }\end{array}$ & dopant & $\begin{array}{c}\text { dopant } \\
\text { concentration }\end{array}$ & resistivity & $\begin{array}{c}\text { implantation } \\
\text { energy }\end{array}$ & $\begin{array}{l}\text { accumulated } \\
{ }^{56} \mathrm{Mn} \text { fluence }\end{array}$ \\
\hline (i) & $n$ & phosphorus & $\sim 1 \times 10^{15} \mathrm{~cm}^{-3}$ & $3-12 \Omega \mathrm{cm}$ & $30 \mathrm{keV}$ & $2.4 \times 10^{13} \mathrm{~cm}^{-2}$ \\
\hline (ii) & $n$ & phosphorus & $\sim 1 \times 10^{15} \mathrm{~cm}^{-3}$ & $3-12 \Omega \mathrm{cm}$ & $60 \mathrm{keV}$ & $1.5 \times 10^{13} \mathrm{~cm}^{-2}$ \\
\hline (iii) & $n^{+}$ & arsenic & $\sim 3 \times 10^{19} \mathrm{~cm}^{-3}$ & $(1-5) \times 10^{-3} \Omega \mathrm{cm}$ & $30 \mathrm{keV}$ & $2.5 \times 10^{13} \mathrm{~cm}^{-2}$ \\
\hline (iv) & $p^{+}$ & boron & $\sim 2 \times 10^{19} \mathrm{~cm}^{-3}$ & $(4.8-5.8) \times 10^{-3} \Omega \mathrm{cm}$ & $30 \mathrm{keV}$ & $3.6 \times 10^{13} \mathrm{~cm}^{-2}$ \\
\hline
\end{tabular}

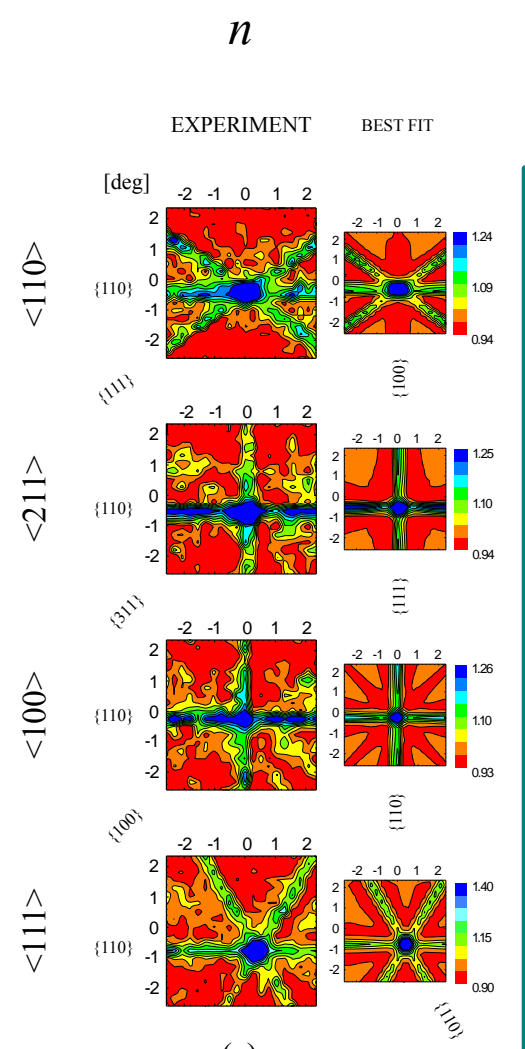

(a) $n$

EXPERIMENT BEST FIT

EXPERIMENT

BEST FIT
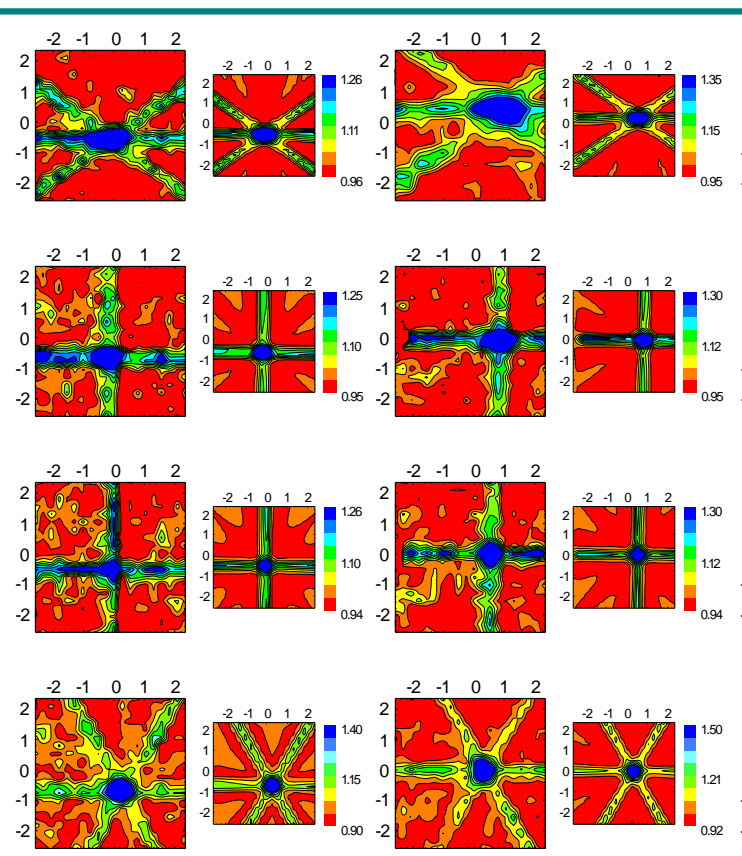

(b)
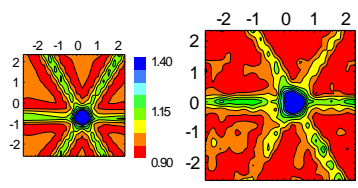

(c)

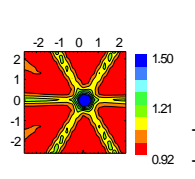

$30 \mathrm{keV}$

$60 \mathrm{keV}$

$p^{+}$

EXPERIMENT BEST FIT
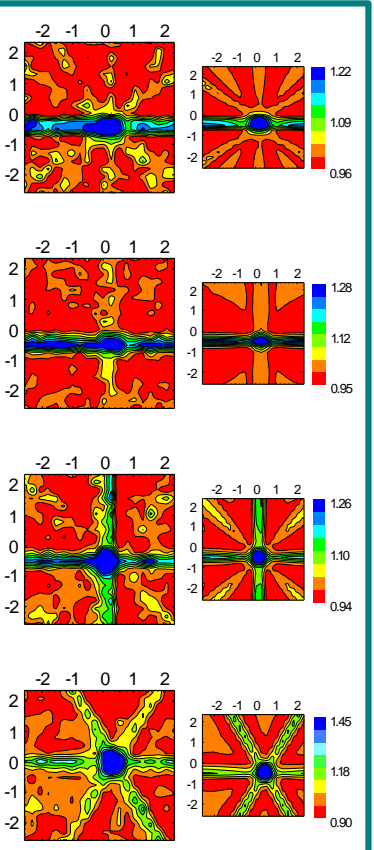

(d)

FIG. 1. Two-dimensional experimental and calculated emission channeling patterns from ${ }^{56} \mathrm{Mn}$ in $(\mathbf{a}, \mathbf{b}) n$-type, $(\mathbf{c}) n^{+}$-type and (d) $p^{+}$-type Si, after annealing at $200^{\circ} \mathrm{C}$. While in $(\mathbf{a}){ }^{56} \mathrm{Mn}$ was implanted at $60 \mathrm{keV}$, in (b-d) the implantation energy was $30 \mathrm{keV}$.

site). For this sample, long-range diffusion seems only to start above $500^{\circ} \mathrm{C}$.

\section{C. $p^{+}$-type}

In experiment (iv), similar fractions for the three identified lattice sites of ${ }^{56} \mathrm{Mn}$ are again observed at room temperature. However, once the annealing temperature increases one notices that both near-BC and near- $\mathrm{AB}$ fractions differ from those of ideal S sites, with a small, but consistent, prevailing of the near- $\mathrm{AB}$ sites. Both near- $\mathrm{BC}$ and near- $\mathrm{AB}$ sites show the highest displacements compared to $n$ - and $n^{+}-\mathrm{Si}$, as observed in Fig. 3: near-AB and near-BC sites are displaced $\sim 0.5 \AA$ from the ideal $\mathrm{AB}$ site and ideal $\mathrm{BC}$ site, respectively. As for the case of the lightly $n$-type doped $\mathrm{Si}$ samples, the sum fraction begins to reduce already at $300^{\circ} \mathrm{C}$, suggesting long-range diffusion of $\mathrm{Mn}$. 


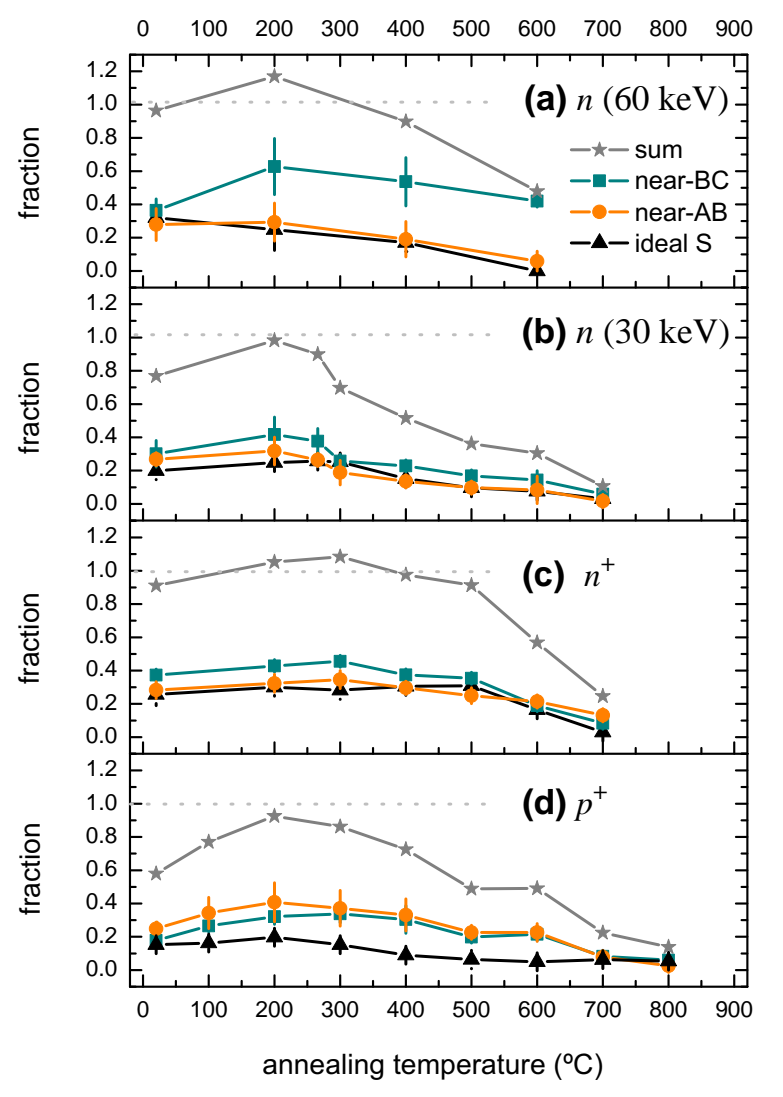

FIG. 2. Fractions of the three identified lattice sites as a function of annealing temperature for (a) n-type Si after implanting at $60 \mathrm{keV}$, and (b) $n$-type, (c) $n^{+}$-type and (d) $p^{+}$-type $\mathrm{Si}$, after implanting at $30 \mathrm{keV}$.

\section{DISCUSSION}

The observation of the non regular sites near-BC and near-AB sites suggests the involvement of complex structures. Moreover, isolated interstitial $\mathrm{Mn}_{\mathrm{i}}$ may quite likely play only an insignificant role since its fast diffusing behavior would allow it to be trapped at other defects already at room temperature. It is possible that ${ }^{56} \mathrm{Mn}$ interacts with contaminant impurities, e.g. O, $\mathrm{H}$ or $\mathrm{C}$. However, using similar arguments as given for EC studies on other $3 \mathrm{~d}$ TMs in $\mathrm{Si}^{17,24-30}$ such as the influence of different silicon materials (Czochralski or float zone silicon) and comparison of both the TM and contaminant impurity concentrations, one can conclude that such interactions, if present, only affect a small fraction of ${ }^{56} \mathrm{Mn}$. Hence, the most plausible origin for the observed lattice sites may come from the interaction of $\mathrm{Mn}$ with self-interstitials, vacancies and electric dopants. While the trapping of $\mathrm{Mn}$ into defective regions of the silicon material (involving vacancies and self-interstitials), may result from local changes in the lattice potential caused by open volumes or strain, the pairing of Mn with elec-

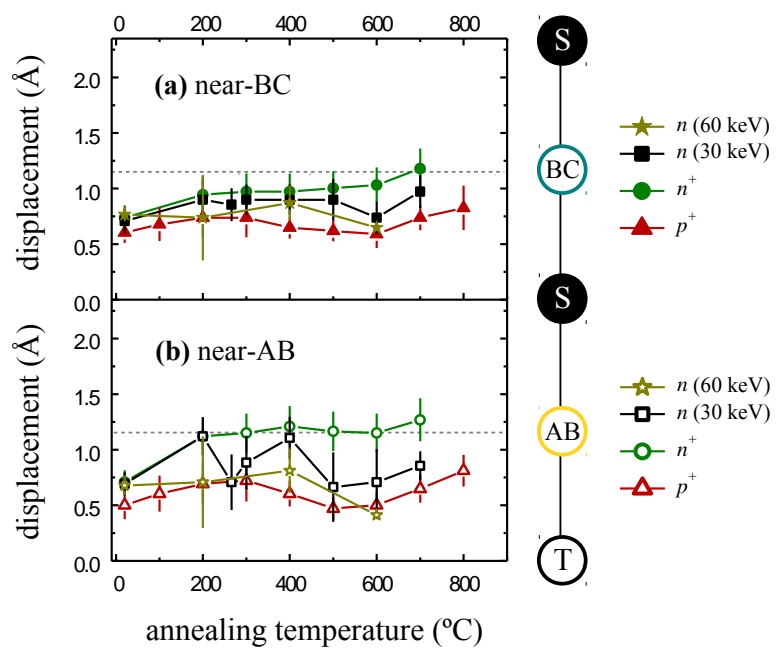

FIG. 3. Displacements of the (a) near-BC and (b) near-AB sites from $\mathrm{S}$ towards $\mathrm{BC}$ sites and from $\mathrm{T}$ towards $\mathrm{AB}$ sites, respectively, as a function of annealing temperature, for all the three types of $\mathrm{Si}$ and two different implantation energies.

tric dopants is mostly driven by the Coulomb interaction, e.g. immobile $\mathrm{B}_{\mathrm{S}}^{-}$will attract $\mathrm{Mn}_{\mathrm{i}}^{+}$. The charge state of the fast diffusing interstitial $\mathrm{Mn}_{\mathrm{i}}$ will hence determine the ease of Mn-dopant pair formation. In that respect, it has been found consistently the existence of one deep acceptor level at $\sim E_{C^{-}}-0.45 \mathrm{eV} \cdot{ }^{10,11}$ Additionally, although still controversial, two detected deep levels (one at $\sim E_{C^{-}}$ $0.12 \mathrm{eV}$ and another at $\sim E_{V}+0.32 \mathrm{eV}$ ) have also been assigned to the interstitial form of Mn. ${ }^{10}$ Therefore, while in $n^{+}$-type $\mathrm{Si} \mathrm{Mn}$ is neutral (or negatively charged), in $p^{+}$-type $\mathrm{Si} \mathrm{Mn}$ is mostly positively charged. Another important phenomenon that could play a role here are possible band-bending and Fermi level pinning effects near the Si surface. In Ref. 31 it is described how interface states created by ion implantation can result, even in highly doped samples, in a Fermi level that is effectively pinned near mid gap position close to the interface between $\mathrm{Si}$ and its surface oxide. Such behaviour might in particular explain the fact that following $30 \mathrm{keV}$ implantation quite similar lattice site distributions are observed for different doping types of $\mathrm{Si}$. In that respect, we should note that all samples were implanted as received from the manufacturer without any treatment to remove the native oxide. In fact, when compared to previous emission channeling investigations on $\mathrm{Fe}, \mathrm{Ni}$ and $\mathrm{Co}$, where marked differences were detected between different doping types, ${ }^{24,29,30}$ here a less pronounced influence on the lattice sites is observed, which may have its origin in the depth profile of $\mathrm{Mn}$ implanted at $30 \mathrm{keV}$ being located closer to the surface and possibly partially in a region of Fermi level pinning. Last but not least, aggregation of Mn may also occur. However, clustering has been ruled out in an EC study on $\mathrm{Fe},{ }^{28}$ where different fluences of 
Fe (between $10^{12}$ and $10^{14} \mathrm{~cm}^{-2}$ ) showed essentially the same behavior. There is no reason to expect Mn to behave differently. In the following, it is discussed in detail possible origins for the observed three lattice sites as well as their thermal stabilities.

\section{A. Ideal $\mathbf{S}$ sites}

The incorporation of $\mathrm{Mn}$ on ideal $\mathrm{S}$ sites most likely results from its trapping into the single vacancies produced during implantation. Ab initio calculations predict a small expansion (1\%) of the silicon lattice around the substitutional Mn. ${ }^{32}$ However, the emission channeling technique is not sensitive to ideal breathing mode relaxations that leave the radioactive probe atom centered on an ideal lattice site, so we can not infer such effects from our measurements. The incorporation of $\mathrm{Mn}$ on ideal $\mathrm{S}$ sites seems to be hampered by the existence of more effective traps. This is likely to be more problematic when creating more defects, allowing their aggregation, e.g. by increasing the fluence. By comparing to $\mathrm{Co}$, which is also a potential magnetic dopant in $\mathrm{Si}$, one can observe that the substitutional fraction of $\mathrm{Co}$ can reach twice that of the present case of $\mathrm{Mn}$, for certain annealing treatments. ${ }^{30}$ One crucial observation for the understanding of the microscopic nature of the magnetism of $\mathrm{Mn}$ is the fact that the fraction of the ideal $\mathrm{S}$ sites is less than $\sim 30 \%$ in all the studied samples. With $\lesssim 30 \%$ of $\mathrm{Mn}$ on ideal $\mathrm{S}$ sites, the risk of having part of the remaining $\gtrsim 70 \%$ forming other complexes that can destroy the ferromagnetic order of dilute substitutional $\mathrm{Mn}$, is very high. How this can affect the ferromagnetism is not known and, therefore, needs further theoretical investigation that includes structures where Mn occupies near-BC and near-AB sites, discussed in the next two subsections. Finally, one should discuss the fact that the observed ideal $\mathrm{S}$ sites are more thermally stable in $n^{+}$ Si, as observed in Fig. 2 (c). Such fact may be related to some extent to the existence of the deep donor level of the substitutional form of $\mathrm{Mn}$ at $E_{C^{-}} 0.51 \mathrm{eV} .{ }^{10}$ The change of the Fermi level towards the conduction band, from $n$ - to $n^{+}$-type $\mathrm{Si}$, may allow $\mathrm{Mn}$ to change its charge state from + to 0 , reducing the number of conduction electrons of the silicon material. A consequence of this might hence be the increase of the ideal $\mathrm{S}$ fraction in $n^{+}$$\mathrm{Si}$. This phenomenon is similar to the so-called counterdoping, well known for Fe. ${ }^{29}$ Another explanation for the observed more thermally stable substitutional Mn comes from the possible formation of $\mathrm{Mn}^{-} \mathrm{As}^{+}$pairs. In fact, Zhu et al. proposed in 2008 a model where the substitutional incorporation of $\mathrm{Mn}$ in $\mathrm{Si}$ is enhanced by doping with $n$-type impurities, such as As. ${ }^{33,34}$ The mechanism they proposed consisted of the formation of MnAs pairs where Mn occupies substitutional sites and its magnetic moment is not affected.

\section{B. near-BC sites}

Near-BC sites have been identified as one of the most frequently occupied lattice sites of other $3 \mathrm{~d}$ TMs in $\mathrm{Si}^{24,27,29}$ Several origins have been proposed, however all involving the trapping of the TM into multivacancy defects. One suggested mechanism was the trapping of the TMs into the center of double vacancies. The resulting lattice site of the TM would be, however, a perfect BC site. This has been suggested, e.g., for several TMs in Ge. ${ }^{35,36}$ Because this ideal configuration is not observed for $3 \mathrm{~d}$ TMs in $\mathrm{Si}$, it has been proposed that other multivacancy complexes could be involved. In particular, the TM may occupy displaced BC sites inside hexavacancy rings to satisfy the fourfold coordination of neighbor Si host atoms. From Fig. 3, one can observe that the displacement decreases from $p^{+}$to $n$ - and further to $n^{+}$-type Si. A possible explanation could be the more relaxed multivacancy-related structure in $p^{+}$than in $n^{+}$-Si. One intriguing observation that can be made from Figs. 2 (a) to (b) is the increase of the near-BC fraction from the $30 \mathrm{keV}$ to the $60 \mathrm{keV}$ experiments. Two causes can explain such a change. First, although the vacancy concentrations as a function of depth are similar in both cases (in particular near the end of range), in the $60 \mathrm{keV}$ case the absolute number of vacancies is twice as high as at $30 \mathrm{keV}$. Since single vacancies are mobile at $\sim 200^{\circ} \mathrm{C}$, the number of vacancy clusters, which are involved in the complexes where $\mathrm{Mn}$ occupy near-BC sites, will be larger after the $60 \mathrm{keV}$ implantation than after the $30 \mathrm{keV}$ implantation. Second, the experimental patterns of both 60 and $30 \mathrm{keV}$ implanted experiments look rather similar after $200^{\circ} \mathrm{C}$ annealing. Also, the fact that the sum of the fractions fitted for different lattice sites reaches $\sim 115 \%$ following annealing at $200^{\circ} \mathrm{C}$ could be a consequence of some shift of the Mn depth profile towards the surface. While the major influence of such a shift would be a general increase of channeling anisotropy, intensifying the patterns from all lattice sites in a similar way, there are also more subtle changes in the shapes of the patterns, that affect some sites slightly more than others. We checked for such a scenario by using $30 \mathrm{keV}$ Mn depth profiles when fitting the data from the $60 \mathrm{keV}$ experiment. This resulted in a reduction of the sum fraction from $115 \%$ to $63 \%$, with $27 \%$ near-BC , $17 \%$ ideal $\mathrm{S}$, and $19 \%$ near-AB. Hence, a shift of the Mn profile towards the surface would cause the fitted fraction on near-BC sites to be somewhat closer to the fractions on the other two sites. Establishing the exact cause would hence require more systematic studies where implanted Mn fluence and depth profiles are systematically varied. Finally, one should discuss the dependence of the thermal stability of near-BC sites on the doping. The trend is far less clear compared to previous works on $\mathrm{Cu},{ }^{25} \mathrm{Fe}^{17,29}$ and $\mathrm{Ni}^{24}$ Nevertheless, an increase in thermal stability from $n$ to $n^{+}$for the $30 \mathrm{keV}$ experiments seems plausible since in $n$-Si the near-BC fraction starts to decrease significantly already at $T_{A}=300^{\circ} \mathrm{C}$, while in $n^{+}$-Si such 
decrease begins after annealing at $600^{\circ} \mathrm{C}$.

\section{C. near-AB sites}

Before discussing the possible complexes involved in the observed near-AB sites one should first examine the similarities and differences of the mathematical position in the diamond lattice between the near-AB sites found in this work and the near-T sites found in the $\mathrm{Fe}, \mathrm{Ni}$ and $\mathrm{Co}$ emission channeling experiments from Refs. 24, 29, and 30 , respectively. The displacement from the ideal $\mathrm{T}$ site varied considerably across the annealing steps and for different doping types for the near- $\mathrm{T}$ sites of $\mathrm{Fe}, \mathrm{Ni}$ and Co. For instance, Ni showed that the displacement was closer to the ideal AB site at low annealing temperatures, but also closer to the ideal $\mathrm{T}$ site at high annealing temperatures. In those studies, the presence of sites closer to the ideal $\mathrm{T}$ site was followed by an increase of the sum fraction. A plausible explanation was associated with the increase of intensity of channeling effects, suggesting that $\mathrm{Mn}$ on such near-T sites was present midway from the peak of the depth profile to the surface $\left(R_{p} / 2\right)$. On the other hand, the sites closer to the ideal AB site seemed to be immobile around the peak concentration of the implanted TMs $\left(R_{p}\right)$. In the present work, the large majority of Mn seems, therefore, to be located at $R_{p}$ in $n$ - and $n^{+}$-Si samples, since no increase of the sum was observed after the recrystallization at $T_{A} \sim 200^{\circ} \mathrm{C}$. Not much is known in the literature about possible complexes involving near- $\mathrm{AB} /$ near- $\mathrm{T}$ sites, except that interstitial Fe in the neighbourhood of single and double vacancies should be located on metastable, displaced $\mathrm{T}$ sites instead of ideal $\mathrm{T}$ sites like in a perfect lattice. ${ }^{37}$ Because this explanation involves metastable complexes, we suggest that more complicated structures, other than single or double vacancies, are involved. For the $p^{+}-\mathrm{Si}$ experiment, a different scenario has to be considered, since $[\mathrm{Mn}] \sim[\mathrm{B}]$. In fact, as already seen, the pairing of the fast interstitial diffuser $\mathrm{Mn}_{\mathrm{i}}$ with immobile $\mathrm{B}_{\mathrm{s}}^{-}$dopants is driven by Coulomb interactions. ${ }^{38}$ If $\mathrm{Mn}_{\mathrm{i}}$ is positive, the pairing will likely occur. Because $p^{+}$doping shifts the Fermi level towards the valence band, the charge state of $\mathrm{Mn}$ will change from 0 to + , and perhaps further to ++ (see the three deep levels pointed out above). The large majority of $\mathrm{Mn}_{\mathrm{i}}$ will have, therefore, positive charge state, which satisfies an imperative condition for $\mathrm{Mn}_{\mathrm{i}}^{+} \mathrm{B}_{\mathrm{S}}^{-}$pairing. It was suggested that $\mathrm{Mn}$ occupies $\mathrm{T}$ sites while boron keeps its substitutional position within the pair. ${ }^{38}$ In fact, taking into account that such pairing is driven by electrostatic attraction, one can estimate the distance $d$ that would have to result between Mn and
B: $d=q^{2} /\left(4 \pi \epsilon \epsilon_{0} E_{B}\right)$, in which $q$ is the charge of the electron, $\epsilon$ the dielectric constant of $\mathrm{Si}, \epsilon_{0}$ the dielectric constant of vacuum and $E_{B}$ the binding energy of the pair. Assuming $E_{B}=0.5 \mathrm{eV},{ }^{39}$ one obtains $d \sim 2.4 \AA$, which corresponds to a displacement of $0.05 \AA$ from $\mathrm{T}$ towards the ideal $\mathrm{H}$ site (i.e. practically an ideal $\mathrm{T}$ site), if considering that $\mathrm{B}$ is on the ideal $\mathrm{S}$ site. However, the positions closest to ideal $\mathrm{T}$ sites that we observed, were $\sim$ $0.5 \AA$ away in the opposite direction from the ideal T site, towards AB sites. The large displacement from ideal $\mathrm{T}$ positions found in $p^{+}$-type $\mathrm{Si}$ is thus not supporting the simple electrostatic model for the lattice site of $\mathrm{Mn}$ in $\mathrm{MnB}$ pairs. It is hence likely the involvement of several complexes in the observed near-AB sites, in which $\mathrm{MnB}$ pairs are one of them. One argument in favor of this model is related to the higher displacements towards the ideal $\mathrm{T}$ site of near-AB sites from $n$ - to $p^{+}-\mathrm{Si}$, as shown in Fig. 3. One should finally remark that these pairs do not, however, take part in significant quantities when using higher fluences, since [Mn] becomes much larger than [B].

\section{CONCLUSIONS}

We investigated the lattice sites occupied by implanted $\mathrm{Mn}$ in $\mathrm{Si}$, in the highly dilute regime. We found less than $\sim 30 \%$ of $\mathrm{Mn}$ on ideal $\mathrm{S}$ sites. More than $\sim 70 \%$ of $\mathrm{Mn}$ was found to form complex structures with implantation defects, such as with multivacancies. In particular, we found substantial fractions of Mn occupying sites near the bond-centered site and sites near the anti-bonding interstitial site. In $p^{+}$-type $\mathrm{Si}$, the positively charged fast interstitial form of manganese $\mathrm{Mn}_{\mathrm{i}}$ may also pair to $\mathrm{B}_{\mathrm{S}}^{-}$on sites very close to tetrahedral interstitial positions. We attribute the increase of the observed displaced antibonding sites to this pairing phenomenon. These findings illustrate the complex defect physics of transitionmetal doped Si and related dilute magnetic semiconductors, which must be carefully taken into account when investigating their electric and magnetic properties.

\section{ACKNOWLEDGMENTS}

This work was supported by FCT-Portugal, project CERN-FP-123585-2011, the Fund for Scientific Research - Flanders, the KU Leuven Projects No. GOA/2009/006 and GOA/2014/007, and by the European Union FP7through ENSAR, contract 262010. D.J. Silva is thankful for FCT Grant SFRH/BD/69435/2010. Project Norte070124-FEDER-000070 is acknowledged.
1 T. Dietl and H. Ohno, Rev. Mod. Phys. 86, 187 (2014).

2 S. Zhou and H. Schmidt, Materials 3, 5054 (2010).
3 V. Ko, K. L. Teo, T. Liew, T. C. Chong, M. MacKenzie, I. MacLaren, and J. N. Chapman, J. Appl. Phys. 104, 
033912 (2008).

4 R. Leitsmann, C. Panse, F. Küwen, and F. Bechstedt, Phys. Rev. B 80, 104412 (2009).

5 Z. Z. Zhang, B. Partoens, K. Chang, and F. M. Peeters, Phys. Rev. B 77, 155201 (2008).

${ }^{6}$ B. R. Sahu and S. K. Banerjee, Phys. Rev. B 77, 155202 (2008).

7 F. Küwen, R. Leitsmann, and F. Bechstedt, Phys. Rev. B 80, 045203 (2009).

${ }^{8}$ V. Zamudio-Bayer, L. Leppert, K. Hirsch, A. Langenberg, J. Rittmann, M. Kossick, M. Voge, R. Richter, A. Terasaki, T. Möller, B. V. I. S. Kümmel, and J. T. Lau, Phys. Rev. B 88, 115425 (2013).

9 E. R. Weber, Appl. Phys. A 30, 1 (1983).

10 H. Nakashima and K. Hashimoto, J. Appl. Phys. 69, 1440 (1991).

11 T. Roth, P. Rosenits, S. Diez, and S. W. Glunz, J. Appl. Phys. 102, 103716 (2007).

12 S. Zhou, K. Potzger, G. Zhang, A. Mücklich, F. Eichhorn, N. Schell, R. Grötzschel, B. Schmidt, W. Skorupa, M. Helm, J. Fassbender, and D. Geiger, Phys. Rev. B 75, 085203 (2007).

13 S. Zhou, A. Shalimov, K. Potzger, M. Helm, J. Fassbender, and H. Schmidt, Phys. Rev. B 80, 174423 (2009).

14 F. J. Ruess, M. E. Kazzi, L. Czornomaz, P. Mensch, M. Hopstaken, and A. Fuhrer, Appl. Phys. Lett. 102, 082101 (2013).

15 M. M. Otrokov, A. Ernst, V. V. Tugushev, S. Ostanin, P. Buczek, L. M. Sandratskii, G. Fischer, W. Hergert, I. Mertig, V. M. Kuznetsov, and E. V. Chulkov, Phys. Rev. B 84, 144431 (2011).

16 A. Wolska, K. Lawniczak-Jablonska, M. Klepka, M. S. Walczak, and A. Misiuk, Phys. Rev. B 75, 113201 (2007).

17 U. Wahl, J. G. Correia, E. Rita, J. P. Araújo, and J. C. Soares, Phys. Rev. B 72, 014115 (2005).

18 L. M. C. Pereira, U. Wahl, S. Decoster, J. G. Correia, L. M. Amorim, M. R. D. Silva, J. P. Araújo, and A. Vantomme, Phys. Rev. B 84, 125204 (2011).

19 L. M. C. Pereira, U. Wahl, J. G. Correia, S. Decoster, L. M. Amorim, M. R. D. Silva, J. P. Araújo, and A. Vantomme, Phys. Rev. B 86, 195202 (2012).

${ }^{20}$ L. M. C. Pereira, U. Wahl, S. Decoster, J. G. Correia, L. M. Amorim, M. R. D. Silva, J. P. Araújo, and A. Vantomme, Phys. Rev. B 86, 125206 (2012).
21 J. F. Ziegler, Nucl. Instrum. Methods. Phys. Res. Sect. B 219, 1027 (2004).

${ }^{22}$ U. Wahl, J. G. Correia, S. Cardoso, J. G. Marques, A. Vantomme, and G. Langouche, Nucl. Instrum. Methods. Phys. Res. Sect. B 136, 744 (1998).

23 S. Agostinelli and et. al., Nucl. Instrum. Methods Phys. Res. A 506, 250 (2003).

24 D. J. Silva, U. Wahl, J. G. Correia, L. M. C. Pereira, L. M. Amorim, E. Bosne, M. R. da Silva, and J. P. Araújo, J. Appl. Phys. 115, 023504 (2014).

25 U. Wahl, J. G. Correia, A. Vantomme, and G. Langouche, Physica B 273, 367 (1999).

26 U. Wahl, A. Vantomme, G. Langouche, J. P. Araújo, L. Peralta, and J. G. Correia, Appl. Phys. Lett. 77, 2142 (2000).

27 U. Wahl, A. Vantomme, G. Langouche, and J. G. Correia, Phys. Rev. Lett. 84, 1495 (2000).

${ }^{28}$ U. Wahl, J. G. Correia, E. Rita, J. P. Araújo, and J. C. Soares, Nucl. Instrum. Methods Phys. Res. B 253, 167 (2006).

29 D. J. Silva, U. Wahl, J. G. Correia, and J. P. Araújo, J. Appl. Phys. 114, 103503 (2013).

30 D. J. Silva, U. Wahl, J. G. Correia, L. M. C. Pereira, L. M. Amorim, M. R. da Silva, and J. P. Araújo, Semicond. Sci. Tech. 29, 125006 (2014).

31 K. Dev, M. Y. L. Jung, R. Gunawan, R. D. Braatz, and E. G. Seebauer, Phys. Rev. B 68, 195311 (2003).

32 A. AlZahrani, Physica B 405, 4195 (2010).

33 W. Zhu, Z. Zhang, and E. Kaxiras, Phys. Rev. Lett. 100, 027205 (2008).

${ }^{34}$ H. Chen and W. Zhu, Phys. Rev. B 79, 235202 (2009).

35 S. Decoster, S. Cottenier, B. D. Vries, H. Emmerich, U. Wahl, J. G. Correia, and A. Vantomme, Phys. Rev. Lett. 102, 065502 (2009).

36 S. Decoster, S. Cottenier, U. Wahl, J. G. Correia, L. M. C. Pereira, C. Lacasta, M. R. D. Silva, and A. Vantomme, Appl. Phys. Lett. 97, 151914 (2010).

37 S. K. Estreicher, M. Sanati, and N. G. Szwacki, Phys. Rev. B 77, 125214 (2008).

38 D. Gilles, W. Schroter, and W. Bergholz, Phys. Rev. B 41, 5770 (1990).

39 H. Lemke, Phys. Status Solidi A 76, 223 (1983). 